\title{
Central retinal artery occlusion resembling Purtscher-like retinopathy
}

This article was published in the following Dove Press journal:

Clinical Ophthalmology

3 August 20I I

Number of times this article has been viewed

\author{
Takuji Kurimoto' \\ Norio Okamoto ${ }^{2}$ \\ Hidehiro Oku' \\ Yuko Kanbara' \\ Tomohiko Etomi' \\ Masahiro Tonari' \\ Tsunehiko Ikeda' \\ 'Department of Ophthalmology, \\ Osaka Medical College, Takatsuki, \\ Japan; ${ }^{2}$ Okamoto Eye Clinic, Suita, \\ Osaka, Japan
}

Correspondence: Takuji Kurimoto Department of Ophthalmology,

Osaka Medical College,

2-7 Daigaku-machi, Takatsuki,

Osaka 569-8686, Japan

Tel +8I 72683 |22|

Fax +81726818195

Email kuritakul20II20I@yahoo.co.jp

\begin{abstract}
This paper reports three cases of central retinal artery occlusion (CRAO) with Purtscher-like retinopathy and good recovery of visual function. The three cases of CRAO had similar fundus changes, ie, cotton wool patches surrounding the optic disc and whitening of the retina surrounding the fovea with a cherry red spot. Fluorescein angiography showed a delay of arm-to-retina circulation time and a partial defect of choroid circulation. Although the three cases were treated by different regimens of steroid pulse therapy and antiplatelet therapy, visual function recovered well and all disturbances of the retinal and choroid circulations resolved. Although eyes with a CRAO normally have a poor visual prognosis, our three cases responded well to the treatments and recovered good visual function. Thus, cases showing fundus changes similar to our three cases may have a pathogenesis different from that of a complete CRAO.
\end{abstract}

Keywords: central retinal artery occlusion, cotton wool patches, Purtscher retinopathy, steroid therapy

\section{Introduction}

Central retinal artery occlusion (CRAO) leads to severe visual disturbances and is generally resistant to treatment. The fundus changes are characterized by retinal whitening, a cherry red spot, and a pale disc. ${ }^{1}$ The visual prognosis in eyes with CRAO is generally very poor, and the degree of visual damage has been suggested to be related to the duration of retinal ischemia before treatment and occlusion of the retinal ciliary artery. ${ }^{2-6} \mathrm{~A}$ CRAO results from one or more pathological processes, ie, retinal emboli, vessel narrowing and thrombosis, arterial spasm, vascular narrowing caused by extravascular disease, and reduction of blood flow caused by carotid or ophthalmic artery obstruction. However, atypical cases with good visual recovery have been reported. ${ }^{7,8}$ Fundus findings were common in appearance of multiple cotton wool patches and scattered retinal whitening. Hayreh et al investigated fundus findings that appeared frequently in three types of CRAO, ie, the permanent type with or without cilioretinal artery sparing and the transient type defined by the findings of fundus examination and fluorescein angiography. ${ }^{1}$ The more frequent findings were retinal opacity, a cherry red spot, and a pale disc in both transient and permanent CRAO. Only nine (3\%) of 248 eyes showed cotton wool patches, indicating that cotton wool patches are a rare manifestation of CRAO.

Purtscher retinopathy was first described in patients suffering from sudden visual loss after severe head injury, and is characterized by cotton wool patches, retinal whitening, and superficial retinal hemorrhages. Since then, cases with sudden visual loss associated with acute pancreatitis, collagen vascular diseases, and renal failure had 
been reported as having fundus alterations similar to those of Purtscher retinopathy. These cases are considered to be the same entity as Purtscher retinopathy, and are grouped together as Purtscher-like retinopathy. ${ }^{9}$ The pathological mechanism for Purtscher retinopathy remains unknown, but several mechanisms have been hypothesized based on the pathogenesis of more general diseases, eg, occlusion and infarction of the microvascular bed due to small emboli, ${ }^{10,11}$ disseminated intravascular coagulopathy, ${ }^{12}$ and leukocyte aggregation due to complement C5a activation. ${ }^{13,14}$ Raised intrathoracic pressure and venous dilatation have also been suggested to be the cause of Purtscher-like retinopathy. ${ }^{15,16}$ Previously, Gass proposed that Purtscher-like retinopathy is a subtype of CRAO cause by an endogenous emboli. ${ }^{17}$ However, the distinction between atypical CRAO and Purtscher-like retinopathy has not been made.

We present three cases of CRAO with retinal changes resembling Purtscher-like retinopathy, although the exact pathological mechanisms remain unknown. All three cases had good visual recovery after steroid pulse therapy and antiplatelet therapy.

\section{Case report I}

Case 1 was a 55-year-old man who noticed blurred vision in his right eye when he woke and was referred to our hospital on the same day. At initial examination, his best-corrected visual acuity (BCVA) was 0.06 OD and 1.5 OS. The critical flicker frequency was $20 \mathrm{~Hz}$ OD and $36 \mathrm{~Hz}$ OS. A relative afferent pupillary defect was present in his right eye. Fundus examination revealed multiple cotton wool patches surrounding the optic disc and retinal whitening in the macular area of the right eye (Figure 1A). Goldmann perimetry showed a paracentral scotoma and a large absolute scotoma in the lower nasal field (Figure 1E). Fluorescein angiography showed that the arm-toretina circulation time was 33 seconds (normally $\leq 15$ seconds in our clinic), and choroid circulation to the nasal retina was delayed. The areas of occlusion of the retinal arterioles corresponded to the areas where the cotton wool patches were found (Figure 1B). There were no plaques in the arteries or apparent spasm of the retinal vessels (Figure 1B). In the late phase of fluorescein angiography, there was no staining of the retinal vessels (data not shown). No significant stenosis of the carotid artery was found by ultrasound examination (internal carotid artery plaque score 1.7$)$. Systemic examination showed no diseases except for hypertension, that was well controlled by medication, and no traumatic history.

Based on the clinical findings, the case was diagnosed as CRAO combined with ischemic optic neuropathy.
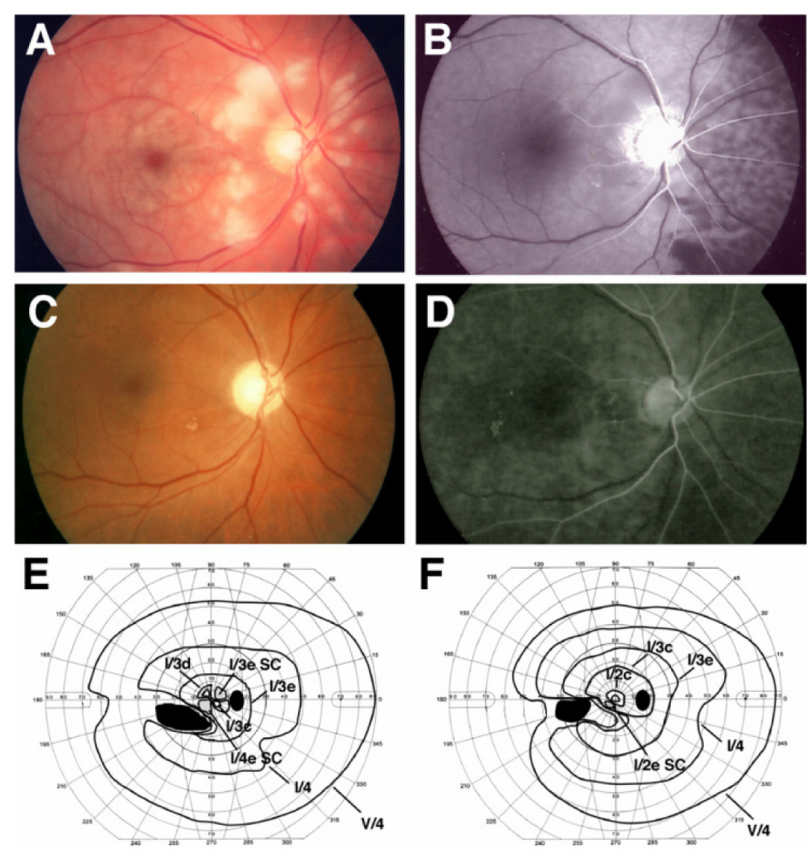

Figure I Clinical findings for Case I. Fundus photographs and fluorescent angiograms before treatment. There are many cotton wool patches surrounding the optic disc and retinal whitening surrounding the fovea $(\mathbf{A})$. Fluorescein angiography shows nonperfused areas of retinal arterioles in the areas corresponding to the areas of cotton wool patches. A delay in the choroidal filling in the nasal area and arm-to-retina time (32 seconds) was found (B). Fundus photograph (C) and fluorescent angiogram (D) after treatment. Optic disc is slightly pale, and defects of the nerve fiber layer can be seen (C). Fluorescein angiography shows improvement of the retinal circulation (D). Arm-to-retina time was 15 seconds. Goldmann kinetic perimetry before $(\mathbf{E})$ and after $(\mathbf{F})$ treatment. There is a large absolute scotoma in the lower nasal field and paracentral scotoma before treatment $(\mathbf{E})$. The paracentral scotoma is decreased after treatment $(\mathbf{F})$.

Note: Black indicates absolute scotoma and gray relative scotoma in $\mathbf{E}$ and $\mathbf{F}$. Abbreviation: SC, scotoma.

An intravenous drip of methylprednisolone (1 $\mathrm{g}$ each day for 3 days), antiplatelets, and vasodilator therapy was started after our initial examination. One month after initiation of therapy, BCVA in the right eye improved to 0.8 , and the retinal lesions were noticeably improved. One month later, the BCVA in the right eye further improved to 1.2 .

\section{Case report 2}

Case 2 was a 47-year-old man who complained of blurred vision in his left eye and came to our hospital 3 days later. At initial examination, the BCVA of both eyes was 1.2, and the intraocular pressure was within the normal range. A relative afferent pupillary defect was not present in his left eye. Fundus examination showed multiple cotton wool patches surrounding the optic disc and slight retinal whitening around the fovea of the left eye (Figure 2A). Fluorescein angiography showed that the arm-to-retina circulation time was 23 seconds, and the areas of occluded retinal capillaries corresponded to the areas of the cotton wool patches (Figure 2B). Staining of the retinal arteries 

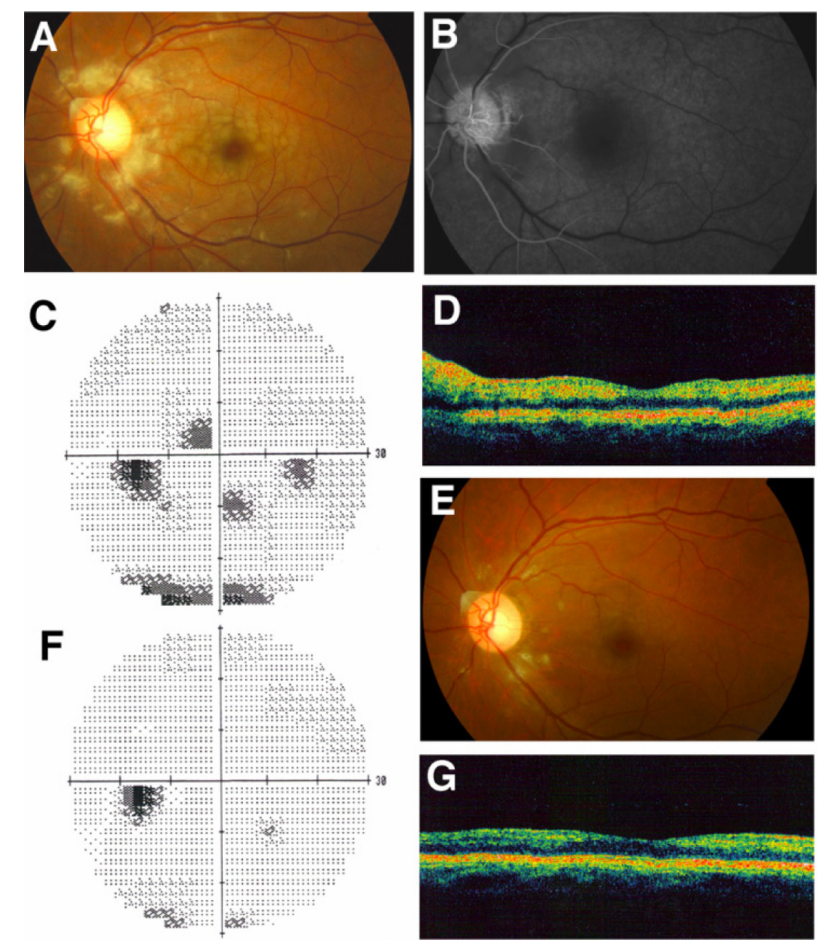

Figure 2 Clinical findings for Case 2. Fundus photographs and fluorescein angiograms before treatment. There are many cotton wool patches surrounding the optic disc and retinal whitening surrounding the fovea $(\mathbf{A})$. Fluorescein angiography showed nonperfused areas of retinal arterioles in areas corresponding to the cotton wool patches. The arm-to-retina time was 28 seconds (B). Humphrey static perimetry at onset $(\mathbf{C})$ and I month later $(\mathbf{F})$. Paracentral scotomas are almost gone. Fundus photograph I month after the onset (E). Most of the cotton wool patches and retinal whitening have disappeared. Optical coherence tomography at onset (D) and I month later (G). Swelling of the inner retina showing hyper-reflectivity was found at onset (D), but the hyper-reflectivity has disappeared and retinal thickness returned to normal I month after onset $(\mathbf{G})$.

was not detected in the late phase of fluorescein angiography (data not shown). Humphrey static perimetry showed several paracentral scotomas (Figure 2C), and optical coherence tomography (Stratus OCT 3000, Humphrey Zeiss) showed a slight thickening of the inner retina but not the retinal ganglion cell layer due to edematous changes (Figure 2D). From the clinical findings, we diagnosed the case as an incomplete CRAO.

Immediately after initial examination, the eye was massaged and acetazolamide was prescribed to reduce the intraocular pressure. The patient was followed up as an outpatient. Three weeks later, the cotton wool patches and retinal whitening disappeared without any medication (Figure 2E), and the thickness of the inner retinal layers was within the normal range in the optical coherence tomography images (Figure 2G). The Humphrey field analyzer findings improved and most of the scotomas were resolved (Figure 2F). On systemic enquiry, there were no systemic diseases except for hypertension that was well controlled with medication. There was no history of trauma.

\section{Case report 3}

Case 3 was a 66-year-old man who noticed decreased vision in his left eye and visited our hospital. At initial examination, his BCVA was $1.2 \mathrm{OD}$ and $0.7 \mathrm{OS}$, and the intraocular pressures were $18 \mathrm{mmHg}$ OD and $14 \mathrm{mmHg}$ OS. No abnormalities were detected in either fundus, and we followed him as an outpatient without any medication. He had hypertension and diabetes mellitus that were well controlled with medication and had no history of traumatic injury.

Four days later, his left BCVA suddenly decreased to 0.4 and the critical flicker frequency was $23 \mathrm{~Hz}$. The direct pupillary light reflex in the left eye was weak and a relative afferent pupillary defect was detected in the left eye. Fundus examination showed cotton wool patches surrounding the optic disc and slight retinal whitening in the macular area (Figure 3A). Fluorescein angiography showed that the armto-retina circulation time was 28 seconds, and the choroidal circulation to the nasal retina was delayed (Figure 3B).
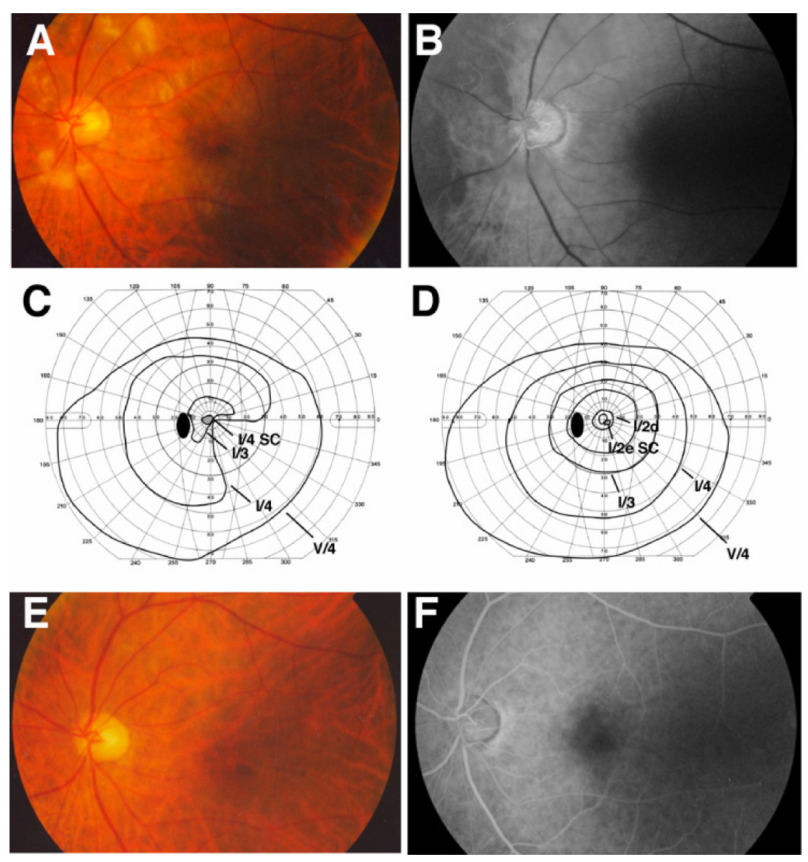

Figure 3 Clinical findings for Case 3. Fundus photographs and fluorescein angiograms before treatment. There are many cotton wool patches surrounding the optic disc and slight retinal whitening surrounding the fovea (A). Fluorescein angiography showing that nonperfused areas of the retinal arterioles were found in the areas corresponding to the cotton wool patches. The delay in choroidal filling in the nasal area and arm-to-retina time (28 seconds) were found (B). Goldmann kinetic perimetry before (C) and 6 weeks after (D) treatment. There is a central scotoma and a constriction in lower nasal field and paracentral scotoma before treatment (C). Central scotoma is decreased and the constriction of lower nasal field has abated after treatment (D). Fundus photograph (C) and fluorescein angiogram (D) 6 weeks after treatment. Cotton wool patches are not present and optic disc is slightly pale (E). Fluorescein angiography showed improvement of retinal circulation 38 seconds after the injection of fluorescein (D).

Note: Black indicates absolute scotoma and gray relative scotoma in $\mathbf{C}$ and $\mathbf{D}$. Abbreviation: SC, scotoma. 
The areas of the occluded retinal capillaries corresponded to the cotton wool patches but there was no staining of the retinal arteries. Goldmann kinetic perimetry showed a central scotoma and a defect in the lower nasal field (Figure 3C). Color Doppler imaging of the cervical artery found no significant stenosis.

Based on the clinical findings, it was believed that the patient had disturbances of both the retinal and choroidal circulations. The RAPD, decreased critical flicker frequency, and central scotoma were related to ischemic optic neuropathy rather than retinal artery occlusion. After diagnosing our patient as having CRAO combined with ischemic optic neuropathy, an intravenous drip of methylprednisolone ( $1 \mathrm{~g}$ each day for 3 days) and antiplatelet therapy was started after the initial examination. Two weeks later, BCVA in his left eye improved to 0.9 and critical flicker frequency to $31 \mathrm{~Hz}$. The central scotoma and lower nasal field defect were almost gone (Figure 3D). Repeat fluorescein angiography showed a marked improvement in both retinal and choroid circulations (Figure 3E and F).

\section{Discussion}

Purtscher retinopathy is a vaso-occlusive retinopathy associated with traumatic injury, acute pancreatitis, renal failure, and vasculitic diseases, such as dermatomyositis or lupus erythematosus. ${ }^{18-24}$ The lesions are characterized by Purtscher flecken that consist of many discrete areas of retinal whitening and cotton wool patches that are retinal microinfarctions at the level of the nerve fiber layer with or without retinal hemorrhages.

We describe three patients who presented with fundus alterations similar to Purtscher retinopathy. All cases had good visual recovery to at least 0.9 , even though disturbances of the proximal retinal and choroid circulation were initially present. The underlying diseases in our cases were only mild hypertension and diabetes mellitus. In cases 1 and 2, relatively large cotton wool patches were found surrounding the optic disc and a whitening of the retina around the fovea accompanied by a cherry red spot. In case 3 , multiple cotton wool patches were detected surrounding the optic disc but the retinal whitening was mild. The fundus changes in our three cases resembled that of Purtscher-like retinopathy but without any traumatic injury or other systemic diseases that have been associated with Purtscher retinopathy (Table 1).

Fluorescein angiography showed that the arm-to-retina circulation time of all cases was delayed, and two of the cases had a filling defect of the choroid circulation which has not been reported in eyes with Purtscher retinopathy.
In the early phase, retinal capillaries were blocked in the areas corresponding to retinal whitening and the cotton wool patches that were seen surrounding the optic disc. These findings suggest the presence of capillary obstructions at more proximal sites than the arterioles; the arterioles had also been obstructed, and the radial peripapillary capillary network might also be disturbed.

Regarding the pathological mechanisms, an experimental study has shown that intermediate $150 \mu \mathrm{m}$ emboli can cause retinal infarctions resembling Purtscher retinopathy. ${ }^{10}$ Another experimental study found that when the central retinal artery is partially obstructed, cotton wool patches appear around the optic disc. ${ }^{25}$ The ophthalmoscopic and fluorescein angiographic findings in all of our cases were characterized by both retinal and choroidal circulation disturbances and multiple cotton wool patches surrounding the optic disc. These are rare ocular findings in CRAO. Our findings suggest that the pathogenesis of rare subtypes of CRAO, such as our cases, involves partial occlusion of the central retinal artery, and the findings are similar to those of Purtscher-like retinopathy, even though there are no more general diseases which can be linked to Purtscher-like retinopathy.

Iwamasa et al described two cases of CRAO with good recovery of vision and visual fields. ${ }^{7}$ Their fundus changes were very similar to that of our cases, ie, multiple cotton wool patches surrounding the optic disc and retinal whitening in the macular area. Fluorescein angiography showed a delay of arm-to-retina circulation time and the retinal capillary nonperfused areas corresponding to the cotton wool patches. These findings are also similar to those of Purtscher-like retinopathy except for the delay in retinal circulation time. Interestingly, the visual fields of two of their cases recovered by 70 days after onset, as did our cases. Thus, it appears that the course of the signs and symptoms is quite different from those in eyes with typical complete CRAO. The resemblance of the clinical course and findings probably indicates similar pathogenesis of these cases.

Several studies have reported that steroid pulse therapy and intravenous administration of steroids has beneficial effects in Purtscher retinopathy. ${ }^{26,27}$ On the other hand, a retrospective study showed that Purtscher retinopathy can recover spontaneously, with an improvement of at least two lines on the Snellen visual acuity chart at 1 year after onset. ${ }^{28}$

Inflammatory reactions can develop secondarily in the nonperfused areas. An optical coherence tomographic study showed subretinal fluid in the nonperfused areas corresponding to the cotton wool patches in a case of Purtscher retinopathy associated with acute pancreatitis. ${ }^{29}$ Likewise, in our case 
2, optical coherence tomography showed a hyper-reflective area in the nerve fiber and retinal ganglion cell layer which probably represented secondary edema. Although we did not examine how effective steroid therapy alone is against retinal edema in CRAO, steroid therapy probably suppressed the inflammation and improved not only the retinal condition but also the optic nerve ischemic condition.

In conclusion, we report three cases of CRAO with fundus appearances resembling Purtscher-like retinopathy. Our findings support the proposal by Gass ${ }^{17}$ that there are subtypes of CRAO, with some cases having a good visual prognosis. Thus, when cases of CRAO with cotton wool patches are encountered, special attention should be paid to the regimen of steroid therapy.

\section{Acknowledgment}

We thank Professor Duco Hamasaki, Bascom Palmer Eye Institute, University of Miami School of Medicine, for editing this manuscript.

\section{Disclosure}

The authors declare that they have no conflicts of interest in this work.

\section{References}

1. Hayreh SS, Zimmerman MB. Fundus changes in central retinal artery occlusion. Retina. 2007;27(3):276-289.

2. Augsburger JJ, Magargal LE. Visual prognosis following treatment of acute central retinal artery obstruction. Br J Ophthalmol. 1980;64(12): 913-917.

3. Brown GC, Shields JA. Cilioretinal arteries and retinal arterial occlusion. Arch Ophthalmol. 1979;97(1):84-92.

4. Brown GC, Magargal LE. Central retinal artery obstruction and visual acuity. Ophthalmology. 1982;89(1):14-19.

5. Yuzurihara D, Iijima $\mathrm{H}$. Visual outcome in central retinal and branch retinal artery occlusion. Jpn J Ophthalmol. 2004;48(5):490-492.

6. Rumelt S, Dorenboim Y, Rehany U. Aggressive systematic treatment for central retinal artery occlusion. Am J Ophthalmol. 1999;128(6): 733-738.

7. Imasawa M, Morimoto T, Iijima $\mathrm{H}$. Recovery of visual field loss due to central retinal artery occlusion. Jpn J Ophthalmol. 2004;48(3):294-299.

8. Mangat HS. Retinal artery occlusion. Surv Ophthalmol. 1995;40(2): 145-156.

9. Agrawal A, McKibbin MA. Purtscher's and Purtscher-like retinopathies: a review. Surv Ophthalmol. 2006;51(2):129-136.

10. Behrens-Baumann W, Scheurer G, Schroer H. Pathogenesis of Purtscher's retinopathy. An experimental study. Graefes Arch Clin Exp Ophthalmol. 1992;230(3):286-291.

11. Castillo BV, Khan AM, Gieser R, et al. Purtscher-like retinopathy and Horner's syndrome following coil embolization of an intracavernous carotid artery aneurysm. Graefes Arch Clin Exp Ophthalmol. 2005;243(1): $60-62$.

12. Tajunisah I, Patel DK, Subrayan V. Purtscher retinopathy as an initial presentation of thrombotic thrombocytopenic purpura. J Thromb Thrombolysis. 2010;30(1):112-113.

13. Shapiro I, Jacob HS. Leukoembolization in ocular vascular occlusion. Ann Ophthalmol. 1982;14(1):60-62. 
14. Jacob HS, Goldstein IM, Shapiro I, et al. Sudden blindness in acute pancreatitis. Possible role of complement-induced retinal leukoembolization. Arch Intern Med. 1981;141(1):134-136.

15. Orzalesi N, Coghe F. Obstructed axoplasmic transport in Purtscher's traumatic retinopathy. Ophthalmologica. 1980;180(1):36-45.

16. Archer DB, Earley OE, Page AB, et al. Traumatic retinal angiopathy associated with wearing of car seat belts. Eye (Lond). 1988;2 (Pt 6): $650-659$.

17. Gass JDM. Obstructive retinal arterial diseases. In: Gass JDM, editor. Stereoscopic Atlas of Macular Diseases, Diagnosis and Treatment. St Louis, MO: Mosby; 1997.

18. Bhan K, Ashiq A, Aralikatti A, et al. The incidence of Purtscher retinopathy in acute pancreatitis. Br J Ophthalmol. 2008;92(1):151-153.

19. Sauer A, Nasica X, Zorn F, et al. Cryoglobulinemia revealed by a Purtscher-like retinopathy. Clin Ophthalmol. 2007;1(4):555-557.

20. Lai WW, Chen AC, Sharma MC, et al. Purtscher-like retinopathy associated with acute renal allograft rejection. Retina. 2005;25(1): 85-87.

21. Carrera CRL, Pierre LM, Medina FMC, et al. Purtscher-like retinopathy associated with acute pancreatitis. Sao Paulo Med J. 2005; 123(6):289-291.
22. Sellami D, Ben Zina Z, Jelliti B, et al. Purtscher-like retinopathy in systemic lupus erythematosus. Two cases. J Fr Ophtalmol. 2002;25(1): 52-55. French.

23. Lim BA, Ang CL. Purtscher-like retinopathy after retrobulbar injection. Ophthalmic Surg Lasers. 2001;32(6):477-478.

24. Power MH, Regillo MC, Custis PH. Thrombotic thrombocytopenic purpura associated with purtscher retinopathy. Arch Ophthalmol. 1997;115(1):128-129.

25. Spertus AD, Slakter JS, Weissman SS, et al. Experimental carotid occlusion: funduscopic and fluorescein angiographic findings. $\mathrm{Br} J$ Ophthalmol. 1984;68(1):47-57.

26. Wang AG, Yen MY, Liu JH. Pathogenesis and neuroprotective treatment in Purtscher's retinopathy. Jpn J Ophthalmol. 1998;42(4):318-322.

27. Atabay C, Kansu T, Nurlu G. Late visual recovery after intravenous methylprednisolone treatment of Purtscher's retinopathy. Ann Ophthalmol. 1993;25(9):330-333.

28. Agrawal A, McKibbin M. Purtscher's retinopathy: epidemiology, clinical features and outcome. Br J Ophthalmol. 2007;91(11):1456-1459.

29. Alasil T, Tokuhara K, Bowes LD, Fan J. Purtscher-like retinopathy: optical coherence tomography and visual field findings. Ophthalmic Surg Lasers Imaging. March 9, 2010. [Epub ahead of print.]
Clinical Ophthalmology

\section{Publish your work in this journal}

Clinical Ophthalmology is an international, peer-reviewed journal covering all subspecialties within ophthalmology. Key topics include: Optometry; Visual science; Pharmacology and drug therapy in eye diseases; Basic Sciences; Primary and Secondary eye care; Patient Safety and Quality of Care Improvements. This journal is indexed on

\section{Dovepress}

PubMed Central and CAS, and is the official journal of The Society of Clinical Ophthalmology (SCO). The manuscript management system is completely online and includes a very quick and fair peer-review system, which is all easy to use. Visit http://www.dovepress.com/ testimonials.php to read real quotes from published authors. 